\title{
The naïve language expert: introduction to the research topic
}

\author{
Jutta L. Mueller ${ }^{1,2} *$ and Claudia Männel ${ }^{2}$ \\ 1 Psycho/Neurolinguistics Group, Institute of Cognitive Science, University of Osnabrück, Osnabrück, Germany \\ 2 Department of Neuropsychology, Max Planck Institute for Human Cognitive and Brain Sciences, Leipzig, Germany \\ ${ }^{*}$ Correspondence: muellerj@cbs.mpg.de \\ Edited by: \\ Manuel Carreiras, Basque Center on Cognition, Brain, and Language, Spain
}

Since the first seminal reports of young infants' abilities to use both acoustic (e.g., Mandel et al., 1994) and statistical cues (e.g., Saffran et al., 1996) to structure, categorize, and memorize linguistic units from their speech input, the quest for capturing infants' abilities and limitations in the discovery of basic elements and regularities in speech has attracted a lot of attention. While many important findings have been unveiled using sophisticated behavioral methods that allow to measure infants' discrimination of familiar vs. unfamiliar speech sounds, the field has gained a new momentum with the advent of techniques, such as event-related brain potentials (ERPs) or functional near-infrared spectroscopy (fNIRS), which allow to measure discrimination even in the absence of overt behavioral responses. After the first excitement about infants' amazing abilities, new challenges have emerged, for example, the question how different input cues interact, how learner variables, such as bilingual language input, contribute to learning mechanisms, or how low-level learning mechanisms contribute to higher-order language learning, such as word learning or sentence comprehension.

The goal of the current Research Topic is to provide a cuttingedge snapshot of this active research field integrating original research papers using both behavioral and neurophysiological techniques with review articles providing ideas for general frameworks capturing those findings.

Three reviews and one methods article offer global and thought-provoking views on basic principles and computational mechanisms that are operative in early language learning. Seidl and Cristia (2012) provide an overview of research on the discovery of allophony vs. phonemic differences in early infancy and discuss mechanisms supporting this distinction. Bortfeld et al. (2013) make a case for using neurophysiological methods, such as ERPs and fNIRS to investigate two factors they consider basic ingredients for early language learning, namely salience and familiarity. Krogh et al. (2012) provide a timely review of statistical learning across modalities and outline different types of constraints and underlying learning mechanisms. Arciuli and Torkildsen (2012) provide evidence for a close interaction between statistical learning and language processing in normal and impaired language acquisition and call for longitudinal research programs shedding light on this relationship.

Two of the original research papers applied neurophysiological methods. Minagawa-Kawai et al. (2013) report an fNIRS study on the emergence of phonotactic abilities in a cross-linguistic sample of infants. The authors report a null-result and discuss potential methodological pitfalls when using fNIRS. Kooijman et al. (2013) used ERPs measured at the age of 7 months as a predictor of later language skills showing the potential sensitivity and meaningfulness of neurophysiological measures with respect to inter-individual differences across language development.

Another set of research articles focuses on the contribution of prosodic information to the perception of sentential structure. Wellmann et al. (2012) evaluate the role of different prosodic boundary cues in German-learning infants' discrimination of coordinate noun phrases, showing that two out of three cues are sufficient for 8-month-olds to solve this task. For the same age, Bernard and Gervain (2012) show that French-learning infants use prosodic prominence and word frequency as signals to word order in an artificial language.

The largest group of papers deals with specific questions related to speech segmentation. Bosch et al. (2013) investigate word segmentation in 6- and 8-month-olds in previously underinvestigated, syllable-timed languages (i.e., Spanish and Catalan) and provide evidence for the early emergence of this ability in monolinguals and bilinguals. For English-learning infants, Thiessen and Erickson (2012) show that this ability emerges even at 5 months if artificial-language units are marked by transitional probabilities and word stress, and that infants' segmentation is guided by transitional probabilities if both information types are placed in conflict. Yurovsky et al. (2012) also study regularities signaling word-like units in child-directed speech, that is, position and onset cues in naming frames. The authors report that in an artificial language either regularity is sufficient to trigger speech segmentation and subsequent word learning in adults. Mintz (2013) is interested in the question when infants are able to segment morphosyntactic endings from verb stems and provides evidence that this happens starting from the first half of the second year of life. Graf Estes (2012) demonstrates that infants at 11 and 17 months recognize words across acoustic variations after successful statistical segmentation and at the older age even apply these generalizations as labels of new objects.

Finally, our Research Topic contains one study which analyzes infant speech production during the second year of life. Yamashita et al. (2013) study English- and Japanese-learning children's phonetic inventory across 15, 20, and 24 months and assume adultlike vocal tract structures to be present by 24 months of age for both languages.

As a compendium of current research efforts in the field of early language learning mechanisms we are confident that this Research Topic offers novel and stimulating ideas for those who are new to the field and would like to get a timely overview as well as for experts who are interested in current developments. 


\section{REFERENCES}

Arciuli, J., and Torkildsen, J. V. (2012). Advancing our understanding of the link between statistical learning and language acquisition: the need for longitudinal data. Front. Psychol. 3:324. doi: 10.3389/fpsyg.2012. 00324

Bernard, C., and Gervain, J. (2012). Prosodic cues to word order: what level of representation? Front. Psychol. 3:451. doi: 10.3389/fpsyg.2012. 00451

Bortfeld, H., Shaw, K., and Depowski, N. (2013). Disentangling the influence of salience and familiarity on infant word learning: methodological advances. Front. Psychol. 4:175. doi: 10.3389/fpsyg. 2013.00175

Bosch, L., Figueras, M., Teixidó, M., and Ramon-Casas, M. (2013). Rapid gains in segmenting fluent speech when words match the rhythmic unit: evidence from infants acquiring syllabletimed languages. Front. Psychol. 4:106. doi: 10.3389/fpsyg.2013. 00106
Graf Estes, K. (2012). Infants generalize representations of statistically segmented words. Front. Psychol. 3:447. doi: 10.3389/fpsyg.2012.00447

Kooijman, V., Junge, C., Johnson, E. K., Hagoort, P., and Cutler, A. (2013). Predictive brain signals of linguistic development. Front. Psychol. 4:25. doi: 10.3389/fpsyg.2013.00025

Krogh, L., Vlach, H. A., and Johnson, S. P. (2012). Statistical learning across development: flexible yet constrained. Front. Psychol. 3:598 doi: 10.3389/fpsyg.2012.00598

Mandel, D. R., Jusczyk, P. W., and Kemler Nelson, D. G. (1994) Does sentential prosody help infants organize and remember speech information. Cognition 53, 155-180.

Minagawa-Kawai, Y., Cristia, A., Long, B., Vendelin, I., Hakuno, Y., Dutat, M., et al. (2013). Insights on NIRS sensitivity from a cross-linguistic study on the emergence of phonological grammar. Front. Psychol. 4:170. doi: 10.3389/fpsyg.2013.00170

Mintz, T. H. (2013). The segmentation of sub-lexical morphemes in english-learning 15-month-olds.
Front. Psychol. 4:24. doi: 10.3389/fpsyg.2013.00024

Saffran, J. R., Aslin, R. N., and Newport, E. L. (1996). Statistical learning by 8-month-old infants. Science 274 1926-1928.

Seidl, A., and Cristia, A. (2012). Infants' learning of phonological status. Front. Psychol. 3:448. doi: 10.3389/fpsyg.2012 00448

Thiessen, E. D., and Erickson, L. C. (2012). Discovering words in fluent speech: the contribution of two kinds of statistical information. Front. Psychol. 3:590. doi: 10.3389/fpsyg.2012.00590

Wellmann, C., Holzgrefe, J. Truckenbrodt, H., Wartenburger, I., and Höhle, B. (2012). How each prosodic boundary cue matters: evidence from german infants. Front. Psychol. 3:580. doi: 10.3389/fpsyg.2012 00580

Yamashita, Y., Nakajima, Y., Ueda, K., Shimada, Y., Hirsh, D., Seno, T., et al. (2013). Acoustic analyses of speech sounds and rhythms in Japanese- and englishlearning infants. Front. Psychol.
4:57. doi: 10.3389/fpsyg.2013. 00057

Yurovsky, D., Yu, C., and Smith, L. B. (2012). Statistical speech segmentation and word learning in parallel: scaffolding from child-directed speech. Front. Psychol. 3:374. doi: 10.3389/fpsyg.2012.00374

Received: 24 July 2013; accepted: 26 July 2013; published online: 20 August 2013.

Citation: Mueller JL and Männel C (2013) The naive language expert: introduction to the research topic. Front. Psychol. 4:526. doi: 10.3389/fpsyg. 2013.00526

This article was submitted to Language Sciences, a section of the journal Frontiers in Psychology.

Copyright () 2013 Mueller and Männel. This is an open-access article distributed under the terms of the Creative Commons Attribution License (CC BY). The use, distribution or reproduction in other forums is permitted, provided the original author(s) or licensor are credited and that the original publication in this journal is cited, in accordance with accepted academic practice. No use, distribution or reproduction is permitted which does not comply with these terms. 